\title{
RAVENNA, IL SUO PORTO E I SUOI ORIZZONTI MEDITERRANEI:L'IMPORTAZIONE DI MATERIALI MARMOREI FRA DINAMICHE COMMERCIALI ED IDEOLOGICHE (V - VI SECOLO).
}

\author{
CLEMENTINA RIZZARDI
}

\author{
UDC: 711.453.4(450.457)"04/05" \\ 730:552.46(450.457)"04/05" \\ Review \\ Manuscript received: 01. 11. 2015. \\ Revised manuscript accepted: 26. 03. 2016. \\ DOI: 10.1484/J.HAM.5.111342
}

\author{
C. Rizzardi \\ Universita degli studi di Bologna \\ Dipartimento di archeologia \\ Piazza san Giovanni in Monte, 2 \\ Bologna, Italia
}

\begin{abstract}
E' noto che il trasferimento della capitale dell'impero romano d'Occidente da Milano a Ravenna, avvenuto nel 402 al tempo dell'Imperatore Onorio, fu dettato anche dalla necessità di instaurare più facili collegamenti con Costantinopoli e con i più importanti centri del Mediterraneo, grazie alla presenza in tale città di un importante porto, che già in epoca romana contava ben 250 navi. Esso dovette svolgere un ruolo fondamentale non solo nello sviluppo di relazioni commerciali con i più importanti centri del Mediterraneo, ma anche nella formazione di quella koinè culturale e artistica, fortemente osmotica, che ha caratterizzato Ravenna sin dalla Tarda Antichità. La grande ricchezza di sculture di marmo proconnesio, riscontrabili tuttora nei monumenti di V-VI secolo o nei Musei della città, riflette stretti collegamenti commerciali specie con la capitale bizantina.
\end{abstract}

Keywords: Ravenna bizantina, relazioni commerciali, manufatti marmorei

E' noto che il primitivo nucleo abitato di Ravenna non solo era a diretto contatto con il mare, ma era felicemente collegato alla terraferma tramite lagune e canali che consentivano facili navigazioni endolagunari e approdi sicuri. La fisionomia di roccaforte inattaccabile dal retroterra e sbocco commerciale e militare grazie a navigazioni fluviali e costiere, contribuirono a fare di Ravenna il maggior emporio marittimo dell'Adriatico e successivamente una città capitale ${ }^{1}$.

E' ormai acclarato che il trasferimento della capitale dell'impero romano d'Occidente da Milano a Ravenna,avvenuto nel 402 al tempo dell'Imperatore Onorio, fu dettato non solo dal timore delle invasioni barbariche e dalla necessità di meglio fronteggiarle, ma anche dalla necessità di instaurare più facili collegamenti con Costantinopoli e con i più importanti centri del Mediterraneo²: Ravenna non solo si trovava sul mare, ma sin dall'epoca romana godeva della presenza di un importante porto, che contava ben 250 navi, come afferma Dione Cassio e che poteva assicurare la difesa dell'Adriatico e del mediterraneo orientale 3 . Il porto Augusteo,a carattere militare, di ancora dubbia localizzazione, dovette coesistere con un altro a carattere commerciale, forse localizzabile nell'area del Mausoleo di Teodorico, ben presto interrato(Jordanes) a causa della subsidenza e sostituito dal portus novus di cui parla Sidonio Apollinare(Ep.I,5):"intravimus Ravennam...quo loci veterem civitatem novumque portum media via Caesaris ambigas utrum connectat an separet"4.

Interventi archeologici e materiali provenienti anche da recenti scavi effettuati nell'area di Classe hanno contribuito a meglio identificare l'impianto portuale, portando alla luce una serie di magazzini, spesso dotati di portici, utilizzati per lo stoccaggio delle merci ${ }^{5}$ e collegati alla città per mezzo di una strada lastricata(Figg.1.2). Intorno al porto dovette poi svilupparsi un notevole abitato,la CIVITAS CLASSIS cinta di mura e provvista anche di edifici religiosi specie nel $\mathrm{V} \mathrm{e}$ VI secolo(Fig.3) ${ }^{6}$.

L'importante funzione commerciale del porto viene sottolineata anche da Procopio che, dopo aver descritto la

\footnotetext{
${ }^{1}$ Circa il sistema fluvio-lagunare e la sua influenza sulle peculiarità e lo sviluppo del territorio ravennate cfr.: S. PATITUCCI, I porti fluviali nell'Italia padana tra antichità e altomedioevo, in Porti, approdi e linee di rotta, Atti Lecce, 1996, Galatina 1998, p. 239-266; A. RONCUZZI, Il territorio di Ravenna nell'antichità, in Ravenna da Capitale imperiale a capitale esarcale, Atti del XVII Congresso Internazionale di studio sull'Alto Medioevo, (Ravenna, 6-11 giugno 2004), Spoleto, 2005, t. I, p. 383-404, tavv. I-XIX; A. PATITUCCI UGGERI, Il sistema fluvio-lagunare, l'insediamento e le difese del territorio ravennate settentrionale(V-VIII secolo), in Ravenna da Capitale imperiale a capitale esarcale, cit. , p. 253-288 in partic.

${ }^{2}$ Circa le motivazioni che dovettero portare al trasferimento della Capitale dell'Impero romano d'Occidente da Milano a Ravenna cfr. F. W. DEICHMANN, Costantinopoli e Ravenna: un confronto, in Corsi di Cultura sull'Arte Ravennate e Bizantina(=CARB), XXIX, 1982, p. 147-148 in partic. ; V. NERI, Verso Ravenna Capitale: Roma, Ravenna e le Residenze Imperiali tardo-antiche, in:G. C. Susini(a cura di), Storia di Ravenna. I. L'Evo antico, Venezia 1990, p. 535-584. ${ }^{3}$ G. Bermond Montanari, L'impianto urbano e i monumenti, in G. C. Susini( a cura di), Storia di Ravenna. I. L'Evo antico, op. cit(n. 2), p. $223-229$. ${ }^{4}$ Circa la problematica dei vari porti che interessarono Ravenna sin dall'Antichità cfr. : F. W. DeIchmAnN, Ravenna Hauptstadt des spätantiken Abendlandes, Kommentar, II, 3. Teil, Stuttgart, 1989, p. 45-49.

${ }^{5}$ A. AUGENTI, Classe. Archeologia di una città scomparsa, in :A. Augenti( a cura di), Classe. Indagini sul potenziale archeologico di una città scomparsa, Bologna, 2011, p. 15-44; IDEM, Città e porti dall'Antichità al Medioevo, Roma, (I ed. 2010), II ed. , Roma, 2013. p. 44; IDEM, Classe:archeologia di una città scomparsa, in: I. Baldini, S. Cosentino (a cura di), Potere e politica nell'età della famiglia teodosiana(395-455), I linguaggi dell'Impero, l'identità dei barbari, Bari 2013, p. 219-234.

${ }^{6}$ Circa la raffigurazione della Civitas Classis nel mosaico parietale di S. Apollinare Nuovo, cfr. : G. Bovini, La raffigurazione della Civitas Classis e dell'imboccatura dell'antico Porto della città nei mosaici di S. Apollinare Nuovo, in: Studi storici, topografici ed archeologici sul "Portus Augusti" di Ravenna e sul territorio classicano, Faenza, 1961, p. 67-86; C. RIZZARDI, Il mosaico a Ravenna. Ideologia ed arte, Bologna, 2011, p. 100 , fig. 80.
} 


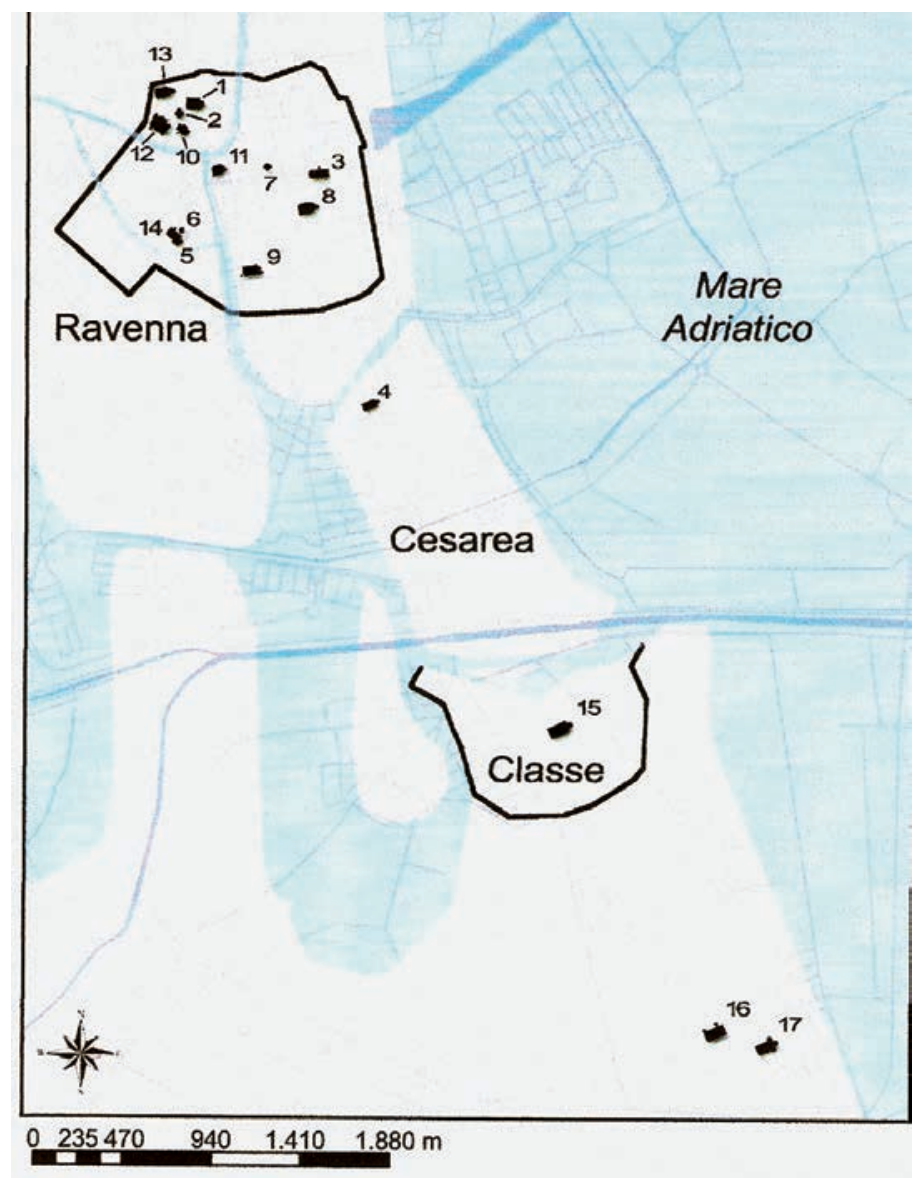

Ravenna, Cesarea, Classe: i monumenti considerati

1. S. Croce
2. Mausoleo di Galla Placidia
3. S. Giovanni Evangelista
4. S. Lorenzo in Cesarea e "Monasterium"
5. dei SS. Stefano, Gervasio e Protasio
6. Battistero Neoniano
7. Battistero degli Ariani
8. S. Apollinare Nuovo
9. S. Agata Maggiore
10. S. Maria Maggiore
11. S. Michele in Africisco
12. S. Vitale
13. S. Stefano Maggiore
14. Basilica Ursiana
15. Basilica Petrian
16. Basilica di S. Apollinare in Classe
17. Basilica Probi

Fig. 1- Ravenna, Cesarea e Classe: i monumenti (da Rizzardi 2011)

difficoltà delle navi ad approdare a causa dell'alta e della bassa marea, così riferisce:"quanti hanno da trasportare derrate in città o da prelevarne per motivo di commercio o altro,posti sulla nave i carichi e tratte queste nel luogo in cui sono solite passare le acque, seguono la corrente. E quando questa giunge, le navi in breve, distaccate da terra, navigano e i marinai occupati viaggiano già" (De bello gothico,5,1,11-17)

Esso dovette svolgere inoltre un ruolo fondamentale nello sviluppo di relazioni commerciali con i più importanti centri del Mediterraneo e ancora nella formazione di quella koinè

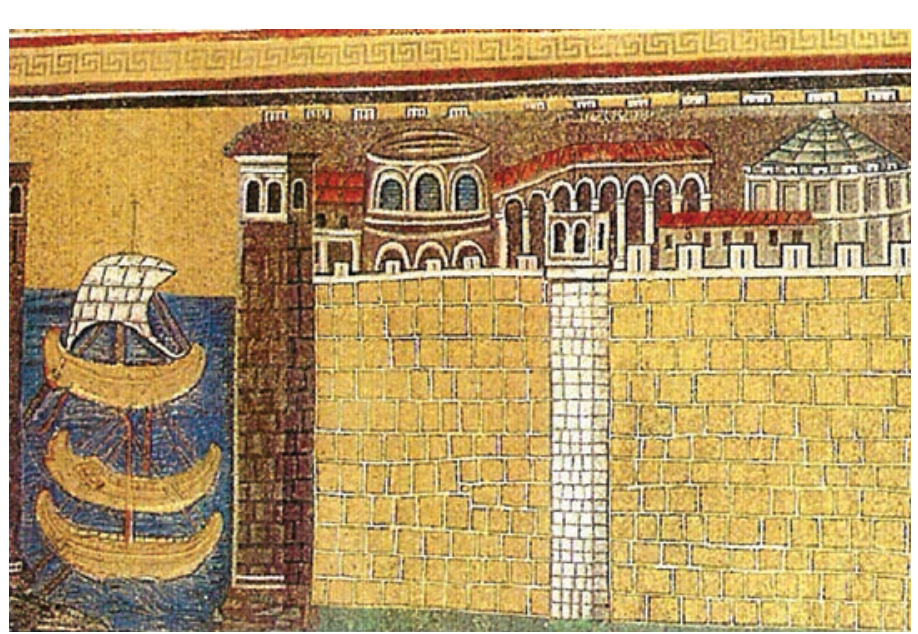

Fig.3-Ravenna, Basilica di S. Apollinare Nuovo, raffigurazione musiva della Civitas Classis (età teodericiana)

culturale e artistica, fortemente osmotica, che ha caratterizzato Ravenna sin dalla Tarda Antichità.

E' ovvio che la presenza della corte esercitò una particolare attrazione sulle principali personalità del tempo e favorì uno straordinario sviluppo urbano, una conseguente fervida attività culturale e una fitta rete di relazioni con i principali centri dell'Impero, che si svilupparono già al tempo di Galla Placidia (425-450) e di Teoderico(493-526), raggiungendo l'apice in epoca giustinianea(540-565).

Attraverso il porto dovettero giungere uomini e merci di ogni tipo, fra cui una grande quantità di sculture di marmo, come si può riscontrare tuttora nei monumenti di V-VI secolo o nei musei della città di Ravenna.

Si tratta soprattutto di sarcofagi che, provenienti in parte da Costantinopoli, ma anche dall'Istria, hanno dato il via ad una gloriosa scuola locale; di colonne marmoree provviste di segni delle officine di fabbrica, di preziose sculture architettoniche o di arredo liturgico(altari,amboni,plutei,transenne,cibori), talora di serie omogenee di elementi(basi, colonne,capitelli,pulvini) di marmo proconnesio prefabbricati per costruzioni ex novo, come ad es. per S.Apollinare in Classe.

Tale straordinario patrimonio culturale- artistico esprime inoltre le linee direttrici di una committenza costituita in prevalenza da imperatori,re, vescovi o arcivescovi che, collegati ideologicamente alla politica imperiale,introdussero soprattutto da Costantinopoli non solo influenze ed idee,ma anche una grande quantità di importanti manufatti marmorei, a testimonianza della loro adesione politica ${ }^{7}$.

Con il progredire delle ricerche,la diffusione dei manufatti di marmo proconnesio nell'ambito dell'Impero ha rivelato dimensioni sempre più ampie che,specie per l'epoca bizantina,comportò implicazioni monopolistiche di carattere politico,sociale,economico ed anche culturale ${ }^{8}$.

7 W. DEICHMANN, Ravenna Geschichte und Monumente, Wiesbaden 1969, p. 63-92; IDEM, Ravenna Hauptstadt, 1989, op. cit, (n. 4), p. 108-115; C. BARSANTI, L'esportazione di marmi dal Proconneso nelle regioni pontiche durante il IV-VI secolo, in Rivista dell'Istituto Nazionale di Archeologia e Storia dell'Arte, a. XII, 1989, Roma, 1990, p. 101-102.

${ }^{8}$ Molto ricca è la bibliografia relativa a questa problematica; fra gli studi più significativi cfr. : F. W. DEICHMANN, Ravenna Geschichte und Monumente, 1969, op. cit. (n. 7), p. 108-115; D. MONNA, P. PENSABENE, P. SODINI, L’identification des marbres:sa nécessitè, ses mèthodes, ses limites, in StMisc, 26, 1985, p. 15-30; C. BARSANTI, art. cit. (n. 7), p. 91-220; R. FARIOLI CAMPANATI, La scultura architettonica e di arredo liturgico a Ravenna alla fine della tarda Antichità: i rapporti con Costantinopoli, in A. Carile (a cura di), Storia di Ravenna, II, 1, Dall'età bizantina alletà ottoniana, Venezia 1992, p. 249-267; J. P. SODINI , C. BARSANTI, A. GUIGLIA GUIDOBALDI, La sculpture architecturale en marbre au VIe siècle à Constantinople et dans les régions sous influence constantinopolitaine, Acta XIII Congressus Internationalis Archeologiae Christianae, (Split-Poreć 25 1994), II, Città del Vaticano-Split, 1998, p. 301-376; R. Farioli Campanati, Ravenna-Costantinopoli:la scultura(secc. V-VI), in:De Luca Ed. (a cura di), Konstantinopel. Scultura bizantina dai Musei di Berlino, Verona, 200o, p. 19-29; EADEM, Ravenna e i suoi rapporti con Costantinopoli: la scultura(secoli V-VI), in: C. RIZZARDI (a cura di), Venezia e Bisanzio. Aspetti della cultura artistica bizantina da Ravenna a Venezia, Istituto Veneto di Scienze, Lettere e Arti, Venezia 2005, p. 13-43; P. PENSABENE, C. BARSANTI, Reimpiego e importazioni di marmi nell'Adriatico paleocristiano e bizantino, in:G. Cuscito( a cura di), Antichita' Altoadriatiche, LXVI, La 


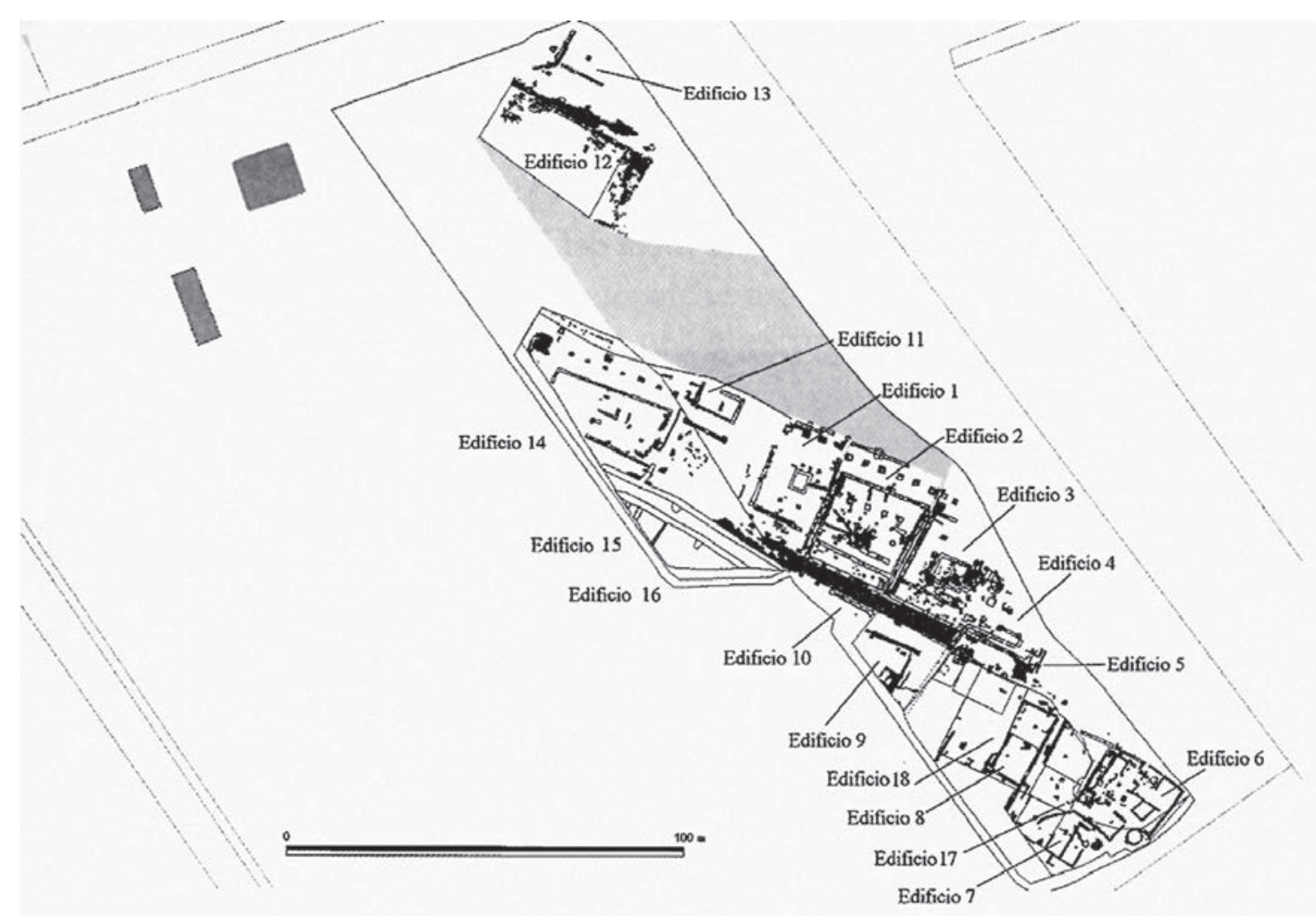

Fig.2-Classe, quartiere portuale, alla luce dei recenti scavi (da Augenti 2011)

E' indubbio che, nell'ambito di tale macroscopico processo di importazione,per quanto concerne Ravenna, il porto di Classe dovette svolgere un ruolo particolarmente significativo: attraverso di esso poterono giungere con facilità ingenti quantitativi di merci, fra cui i preziosi marmi del Proconneso, attuale isola di Marmara,che tuttora arricchiscono il patrimonio artistico della città.

Tali cave,di proprietà imperiale, ma affidate ad appaltatori privati ${ }^{9}$, erano produttrici di un'enorme quantità di marmi bianchi e biancastri di buona qualità e di costo limitatocome emerge già dall'Edictum de Pretiis di Diocleziano- destinato all'esportazione e alla commercializzazione, favorite indubbiamente dalla posizione geografica dell'isola stessa, situata in prossimità dell'imboccatura della Propontide(Mar di Marmara) e dal fatto che le cave si trovavano vicine ad un porto $^{10}$.Il rinvenimento di una grande quantità di manufatti marmorei in diversi stadi di lavorazione,i numerosi studi fatti su di essi, ci hanno fornito dati più concreti per valutare l'enorme sfruttamento di tali cave, sull'attività dei relativi opifici in età romana e bizantina, sui metodi di estrazione, sulle tecniche di lavorazione, sull'organizzazione del lavoro nelle cave e nei relativi opifici, sui sistemi di trasporto nonché sul commercio dei marmi.

Il ben noto e pluricitato recupero di un carico della nave naufragata presso Marzamemi , sulla costa orientale della Sicilia,a nord di Capo Passero (Siracusa) ha potuto note- volmente chiarire le modalità e le proporzioni raggiunte dal trasporto di pezzi prefabbricati, destinati a costruzioni ecclesiastiche, specie in età giustinianea ${ }^{\text {II }}$. Si tratta di una serie quasi completa di elementi marmorei per l'arredo architettonico e liturgico di una basilica, comprendente 28 colonne con relative basi, 28 capitelli corinzi, 12 plutei,dodici pilastrini,12 colonnine, una mensa d'altare in marmo pentelico o microasiatico, 4 sostegni di un ciborio, tutti di marmo proconnesio e tutti rifiniti nella lavorazione, e inoltre 20 pezzi di un ambone di marmo verde di Tessaglia: preziosi manufatti destinati ad una importante basilica, rimasta sconosciuta, eretta dopo la riconquista bizantina in una delle tante località dell'Impero, in linea con il vasto programma di edificazione e di ristrutturazione edilizia promosso da Giustiniano e testimoniato nell'opera De Adificiis di Procopio di Cesarea(I-VI).

Lo studio diretto di molti materiali scultorei conservati a Ravenna, sia di arredo liturgico che di ambito funerario, integra perfettamente i dati provenienti dalle fonti storiche. Priva di cave marmoree locali o della possibilità di usufruire di una considerevole quantità di spolia, la città cominciò a importare marmi sin dall'età placidiana,poi in quantità sempre crescente durante il regno di Teoderico, raggiungendo poi l'apice in epoca giustinianea.

Subito dopo l'avvento di Galla Placidia ${ }^{12}$,nella basilica di San Giovanni Evangelista, da lei fatta innalzare a soluzione

Cristianizzazione dell'Adriatico, Trieste 2008, p. 455-490; Y. A. MARANO, Il commercio del marmo nel Mediterraneo tardo antico (IV-VI secolo). Scambi, maestranze, committenze, Padova, 2008; J. G. HARPER, The Provisioning of Marble for the Sixth-Century Churches of Ravenna:a reconstructive Analysis, in: R. L. Coltella, M. J Gill, L. A. Jenkens, P. Lamers( a cura di), Pratum Romanum. Richard Krautheimer zum 10o. Geburtstag, Wiesbaden, 1997, p. 131-148; A. PARIBENI-E. F. CASTAGNINO BERLINGHIERI, Marble production and marble trade along the Mediterranean coast in Early Byzantine Age (5 $5^{\text {th }} 6^{\text {th }}$ centuries): data from quarries, shipwrecks and monuments, Proceedings of the $15^{\text {th }}$ Symposium on Mediterranean Archaeology(Catania 3-5 March 2011), Oxford 2015, II, pp. 1033-1041

9 Y. MARANO, op. cit, (n. 8), p. 160.

${ }^{10}$ C. BARSANTI, cit. (n. 7), p. 92; circa il prezzo dei marmi vd.: M. GIACCHERO, Edictum Diocletiani et Collegarum de pretiis rerum venalium, Genova 1974, p. 210-211.

${ }^{11}$ G. KAPITÄN, Elementi architettonici per una basilica dal relitto navale del VI secolo di Marzamemi (Siracusa), in CARB, XXVII, 1980, p. 71-136.

${ }^{12}$ Per quanto riguarda l'Augusta Galla Placidia e il suo tempo cfr.: V. A. SIRAGO, Galla Placidia:la Nobilissima(392-450), Milano, 1996; L. STORONI MAZZOLANI, Galla Placidia. Una donna in un'epoca di trapasso, Milano, 2002; P. FUENTES HINOJO, Gala Placidia. Una Soberana del Imperio cristiano, San 


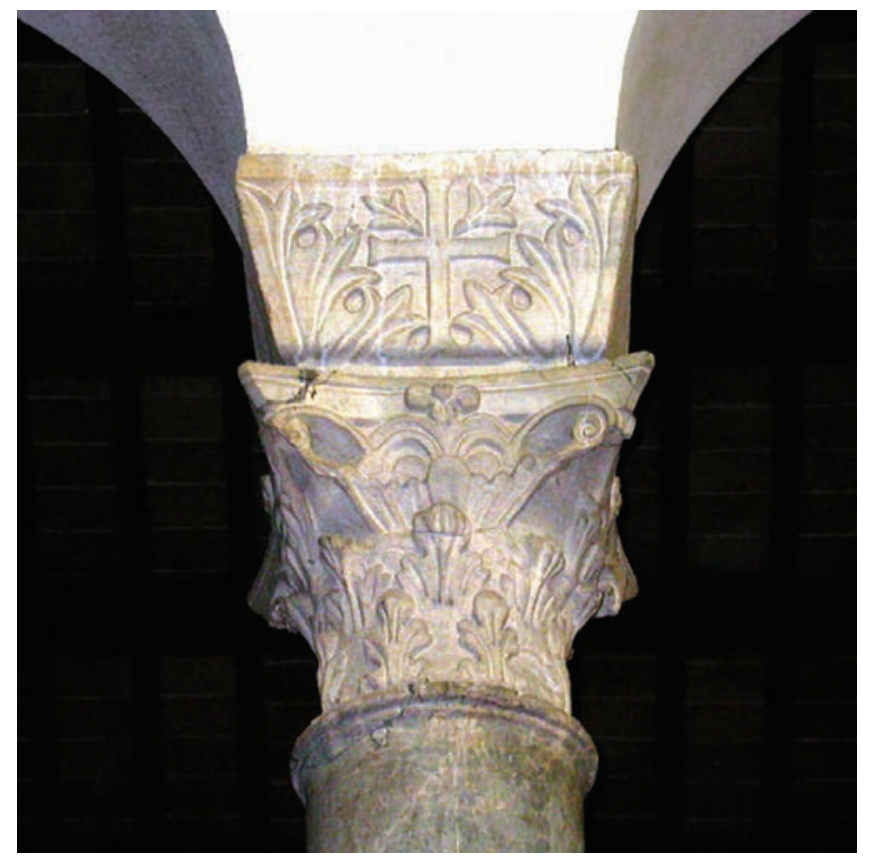

Fig.4-Ravenna, Basilica di San Giovanni Evangelista, capitello e pulvino

di un voto per lo scampato pericolo durante il tentativo di raggiungere via mare Ravenna ${ }^{13}$, degno di rilievo è il fatto che compare per la prima volta un elemento architettonico,ossia il pulvino, ben noto nella parte orientale dell'Impero e che costituisce per l'Occidente un'assoluta novità. Nella basilica ravennate di fondazione imperiale, una serie omogenea di tali manufatti presenta inoltre il tema cristologico-simbolico prediletto in Oriente (Fig.4) ed attestato ad esempio nella basilica di San Giovanni ad Efeso, che si affermerà poi diffusamente in altre classi di scultura ${ }^{14}$.

Un bell'altare a cassa,tuttora esistente al centro del presbiterio di tale basilica(Fig.5), frutto di varie ristrutturazioni,ma originariamente attribuibile all'età placidiana,presenta una grande ricchezza di marmi diversi,dal verde di Tessaglia al giallo antico,al porfido,al marmo grigio e nero,attestando una certa varietà dei marmi importati, prevalentemente, ma non esclusivamente provenienti dal Proconneso ${ }^{15}$.

Un'altra serie di capitelli omogenei, i c.d. capitelli a lira, caratterizza inoltre un edificio di probabile età placidiana:la basilica Apostolorum, attuale basilica di San Francesco ${ }^{16}$; essi rappresentano una tipologia diffusissima in area greco-costantinopolitana fino al VI secolo.

Il fenomeno dell'importazione si estende poi anche alla scultura funeraria,oggetto di un lungo e appassionato dibattito critico,che ha coinvolto non solo il problema della

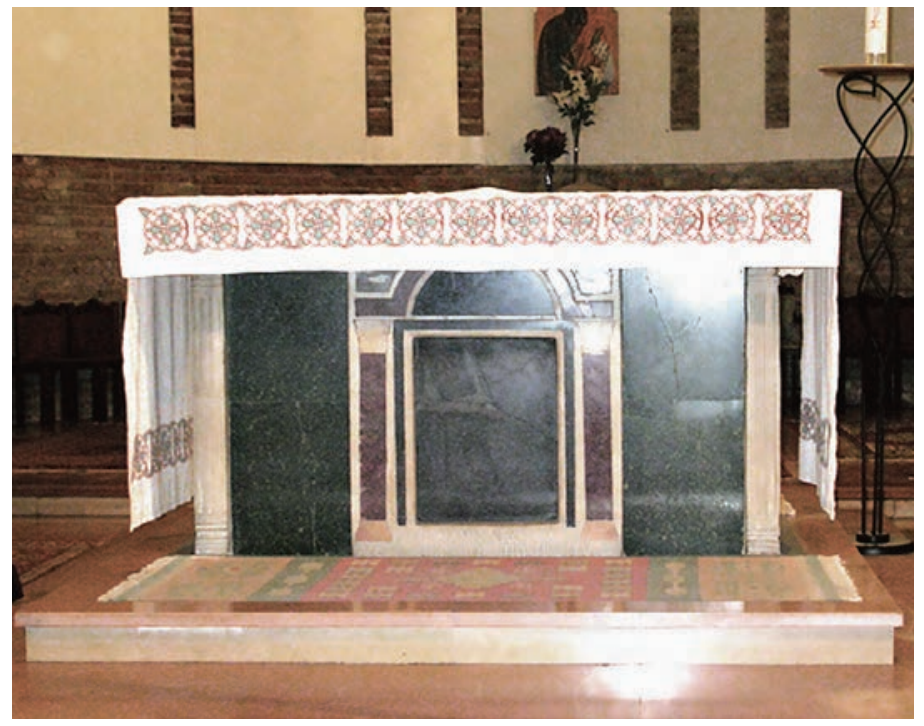

Fig.5-Ravenna, Basilica di San Giovanni Evangelista, altare a cassa

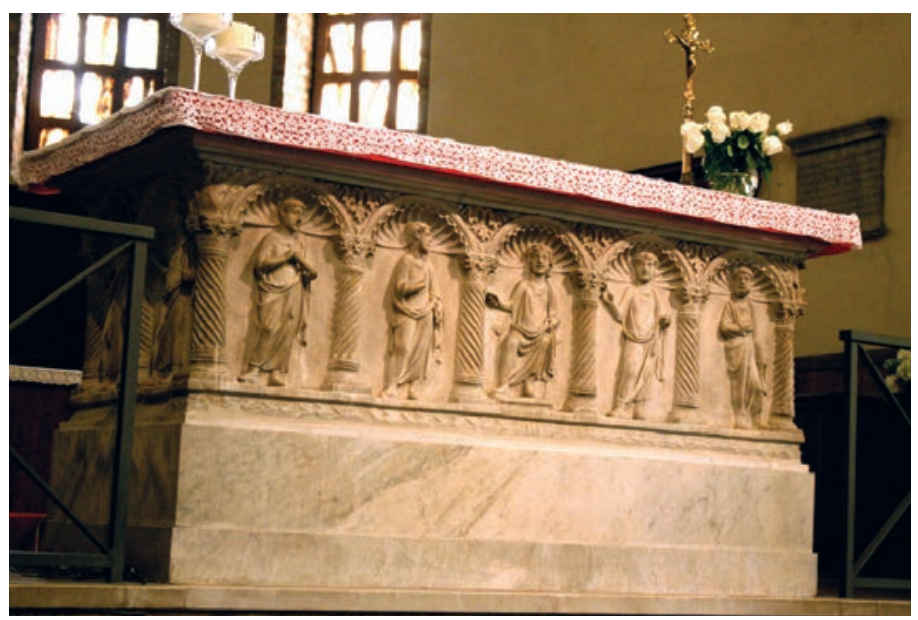

Fig.6-Ravenna, Basilica di San Francesco, sarcofago a nicchie conchigliate, con funzione di altare

cronologia, ma anche della provenienza, delle maestranze,dividendo talora gli studiosi ${ }^{17}$. Di sicura importazione costantinopolitana si devono considerare, alla luce dei più attendibili studi,alcuni sarcofagi, che si porranno poi come modelli per le botteghe ravennati, distinguendosi tuttavia, rispetto alle versioni locali, per la loro nobiltà figurativa, propria di un'arte colta. Si tratta dei due sarcofagi a nicchie conchigliate di San Francesco(fine IV-in.V secolo)(Fig.6), del tutto confrontabili con un frammento rinvenuto dal Deichmann nei depositi del Museo Archeologico di Istanbul, raffigurante la Vergine con Bambino, inserita al di sotto

Sebastian 2004; C. RIZZARDI, Galla Placidia e il suo tempo attraverso la documentazione monumentale e iconografica, in I. Baldini, S. Cosentino (a cura di), Potere e politica nell'età della Famiglia teodosiana (395-455), op. cit. (n. 5), p. 195-218.

${ }^{13}$ F. W. DEICHMANN, Ravenna Hauptstadt des spätantiken Abendlandes, Kommentar, I Teil, Wiesbaden, 1974, p. 93-124; C. RIZZARDI, L'architettura a Ravenna durante il regno di Galla Placidia: problematiche ed influenze artistiche, in Ravenna Studi e Ricerche, 1, 1994, p. 189-202 in partic.

${ }^{14}$ R. FARIOLI, La scultura architettonica, art. cit. (n. 8), p. 251.

${ }^{15}$ L. SOTIRA, Gli altari nella scultura e nei mosaici di Ravenna(V-VIII secolo), Studi e Scavi, 38, Bologna, 2013, n. 1, p. 25-31, figg. 15-18.

${ }^{16}$ R. FARIOLI CAMPANATI, La scultura architettonica, art. cit. (n. 8), p. 251.

${ }^{17}$ Cfr. in particolare:F. W. DEICHMANN, Konstantinopler und ravennatische Sarkophag-probleme, in Bizantinische Zeitschrift, LXII, 1969, p. 291-307; IDEM, Ravenna Geschichte und Monumenta, 1969, op. cit. (n. 8); R. FARIOLI, Il problema delle origini della scultura paleocristiana di Ravenna, in Arheološki vestnik, XXIII, Ljubijana, 1972, p. 167-174; J. KOLLWITZ, H. HERDEJÜRGEN, Die ravennatische Sarkophage, Berlin 1979; R. FARIOLI CAMPANATI, Osservazioni sulla scultura del V- VI secolo:problemi ravennati, in: Passaggio dal mondo antico al Medioevo, Atti del Convegno dell'Accademia dei Lincei, (Roma 25-28 maggio 1977), Roma, 1980, p. 147-194; F. W. DEICHMANN, Ravenna Hauptstadt, 1989, op. cit. (n. 4), p 333-347; P. PORTA, Scultura funeraria paleocristiana tra medio e alto Adriatico: iconografia e simbolismo, in: G. Cuscito (a cura di), Antichità Altoadriatiche LXVI, op. cit. , p. 491-537 (ivi bibl. prec.); per alcune riflessioni sulla scultura funeraria costantinopolitana vd.: J. G. DECKERS, Theodosianische Sepulkralplastik in Konstantinopel. $380-450$ n. Chr. , in:F. Bisconti, H. Brandenburg( a cura di), Sarcofagi tardo antichi, paleocristiani e altomedievali, Città del Vaticano, 2004, p. 35-74. . 


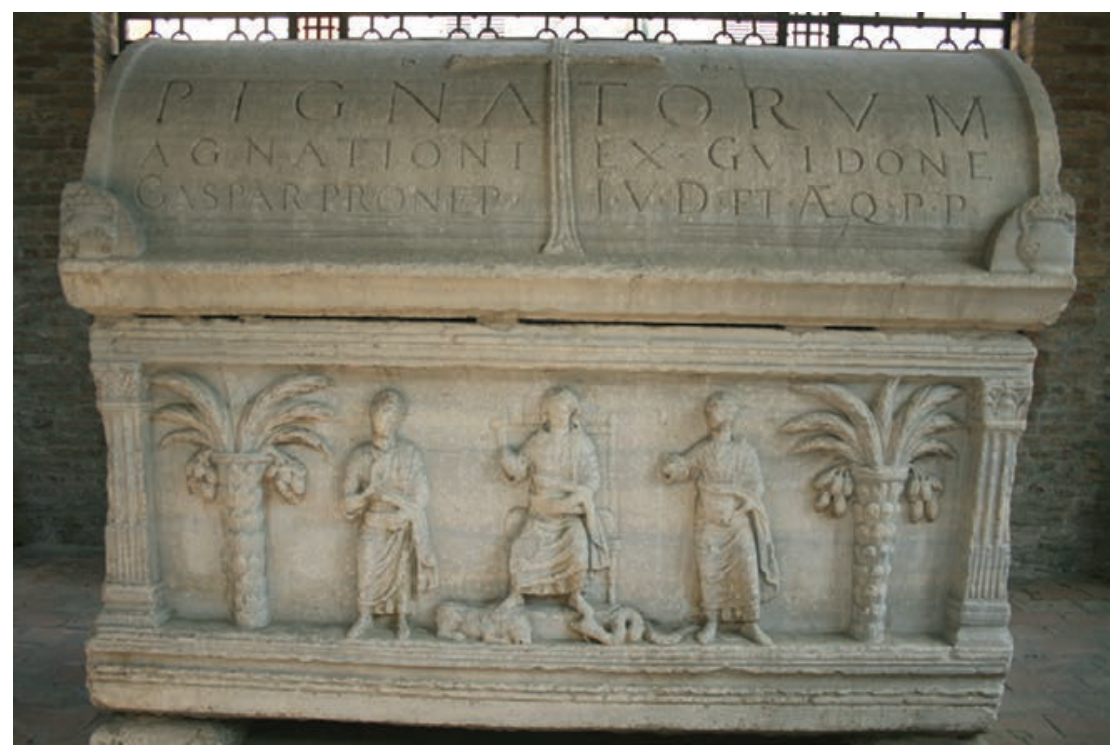

Fig.7-Ravenna, Quadrarco di Braccioforte, Sarcofago Pignatta (foto autore)

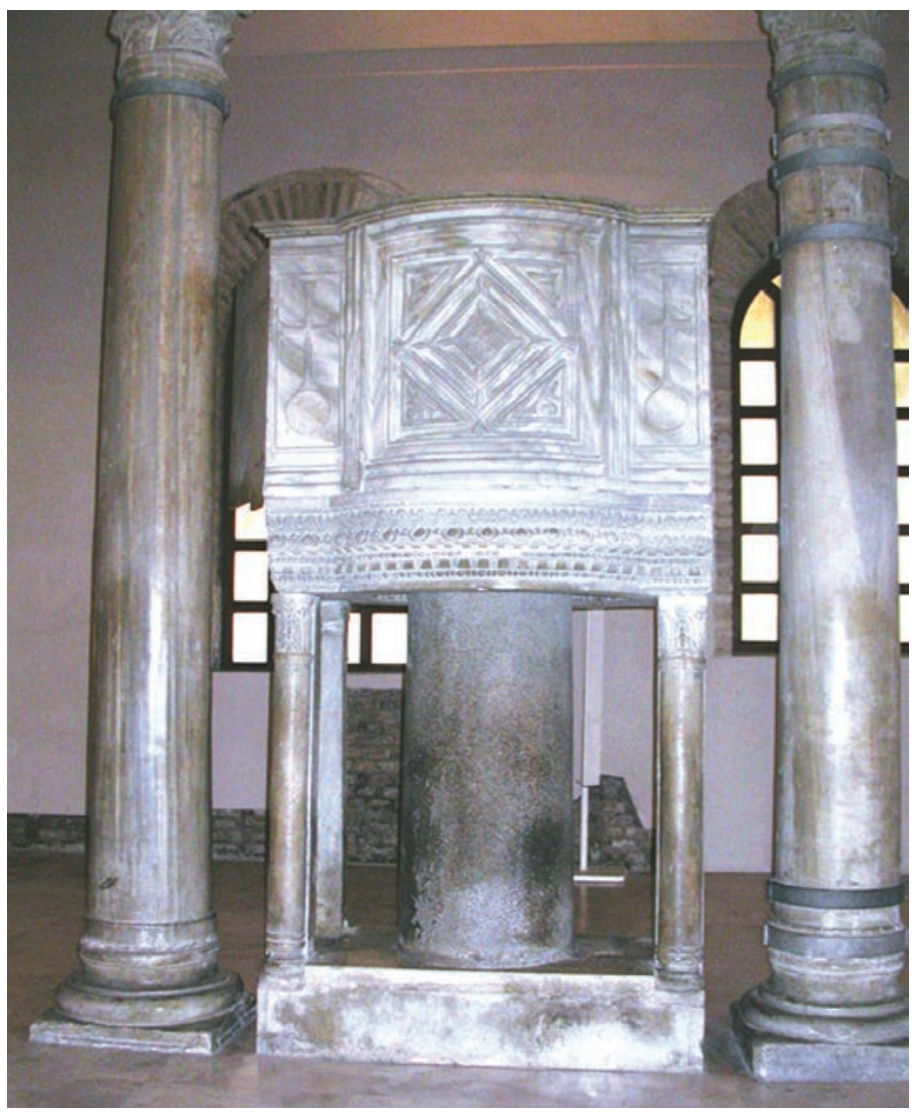

Fig.8-Ravenna,Basilica di Sant'Apollinare Nuovo, ambone di un'identica nicchia conchigliata sorretta da colonnine spiraliformi e da lui ritenuti opera di una stessa bottega attiva a Costantinopoli ${ }^{18}$.Tale osservazione ha dato una svolta praticamente allo studio e alla comprensione dei sarcofagi ravennati,permettendo di distinguere con una certa chiarezza le opere importate da quelle prodotte in loco da maestranze ravennati ${ }^{19}$.

E' possibile inoltre sostenere la provenienza costantinopolitana anche del grandioso sarcofago Pignatta,ubicato nel Quadrarco di Braccioforte (Fig.7),opera di alta qualità artistica ,frutto di quella corrente di tradizione microasiatica che caratterizza la scultura aulica della capitale bizantina $^{20}$.La sua monumentalità, la decorazione su tutti e quattro i lati, il superbo coperchio a botte, la raffinatezza stilistica,permettono di considerarlo un exemplum di scultura funeraria protobizantina, ben diversa da quella occidentale.

Lo stesso si può rilevare anche per quanto concerne il frammento di sarcofago con scena dell'incredulità di S.Tommaso, conservato nel Museo Nazionale di Ravenna(in.V secolo) ${ }^{21}$. Accanto a sculture architettoniche, di arredo liturgico e funerarie, il panorama dei marmi arrivati a Ravenna in concomitanza con il fiorire della corte, si arricchisce anche di sculture ufficiali, come ad es. una statua acefala di clamidato, in porfido,conservata nel Museo Arcivescovile di Ravenna (V secolo), raffigurante un personaggio imperiale vittorioso- non meglio identificabile- nell'atto di deporre la spada nel fodero ${ }^{22}$.

Il legami fra le due capitali si riscontrano, in epoca placidiana, oltre che attraverso l'importazione di sculture, anche per l'introduzione a Ravenna di culti per santi venerati a Costantinopoli e nel vicino Oriente, nonché per le incidenze topografiche e toponomastiche, come ha ben precisato Raffaella Farioli, che sembrano voler imitare la capitale sul Bosforo fondata da Costantino, al quale si richiamano gli Imperatori della nuova dinastia valentiniano-teodosiana, rappresentati anche nei mosaici ora scomparsi della cappella palatina di san Giovanni Evangelista ${ }^{23}$.

Un maggior afflusso di elementi marmorei "prefabbricati” si può rilevare nella successiva età teodericiana(fineV-inizio VI secolo) ${ }^{24}$.Non casualmente la chiesa di S.Apollinare Nuovo, basilica palatina del re goto,è stata dotata, oltre che di un elegante ambone di marmo (Fig.8), che presenta sicuri riscontri con esempi costantinopolitani, di 24 capitelli corinzi "a lira" contrassegnati, al pari di gran parte del fusto delle colonne, da sigle greche di bottega: segni di fabbrica che

${ }_{{ }_{18}}$ F. W. DEICHMANN, art. cit, (n. 17), p. 291-297, fig. 1; R. FARIOLI CAMPANATI, Osservazioni sulla scultura, art. cit (n. 17) , p. 165; a tal proposito cfr. S. PASI, Ravenna e Bisanzio, in:C. Rizzardi( a cura di), Venezia e Bisanzio . Aspetti della cultura artistica bizantina, op. cit. (n. 8), p. 65.

${ }^{19}$ R. FARIOLI, Botteghe ravennati tra Oriente ed Occidente, in: Ravenna da Capitale imperiale a Capitale esarcale, Atti del XVII Congresso Internazionale di studio sull'alto Medioevo (Ravenna, 6-12 giugno 2004), Spoleto, 2005, p. 364-366.

${ }^{20}$ J. KOLLWITZ-H. HERDEJÜRGEN, op. cit. (n. 17), cat. n. B/1, tavv. 24-25; R. FARIOLI CAMPANATI, Osservazioni sulla scultura, art. cit. (n. 17), p. 166; EADEM, Ravenna-Costantinopoli:la scultura, art. cit. (n. 8), p. 22, fig. 5.

${ }^{21}$ J. KOLLWITZ-H. HERDEJÜRGEN, op. cit(n. 17), cat. n. B/2, tav. 26; R. FARIOLI CAMPANATI, Ravenna- Costantinopoli:la scultura architettonica, art. cit. (n. 8), p. 22, fig. 4.

${ }^{22}$ R. FARIOLI CAMPANATI, Ravenna e i suoi rapporti con Costantinopoli, art. cit. (n. 8) , p. 16, fig. 2; A. EFFENBERGER, Torso di statua di Imperatore, in Konstantinopel, cit. (n. 8), p. 40, tav. a p. 41.

${ }^{23}$ Circa le relazioni topografiche ed agiografiche fra Ravenna e Costantinopoli cfr. : R. Farioli Campanati, Ravenna, Costantinopoli:aspetti topografico-monumentali e iconografici, in: A. Carile (a cura di), Storia di Ravenna. II, 2, Dall'età bizantina all'età ottoniana, Venezia 1992, p. 127-157; per i mosaici scomparsi della basilica di San Giovanni Evangelista cfr.: C. RIZZARDI, Il mosaico a Ravenna, op. cit. (n. 6), p. 58-61.

${ }^{24}$ C. BARSANTI, Ravenna:gli arredi architettonici e liturgici negli edifici di età teodericiana, in: C. Barsanti, A. Paribeni, S. Pedone (a cura di), Rex Theodericus. Il medaglione d'oro di Morro d'Alba, Roma 2008, pp. 185-202. 


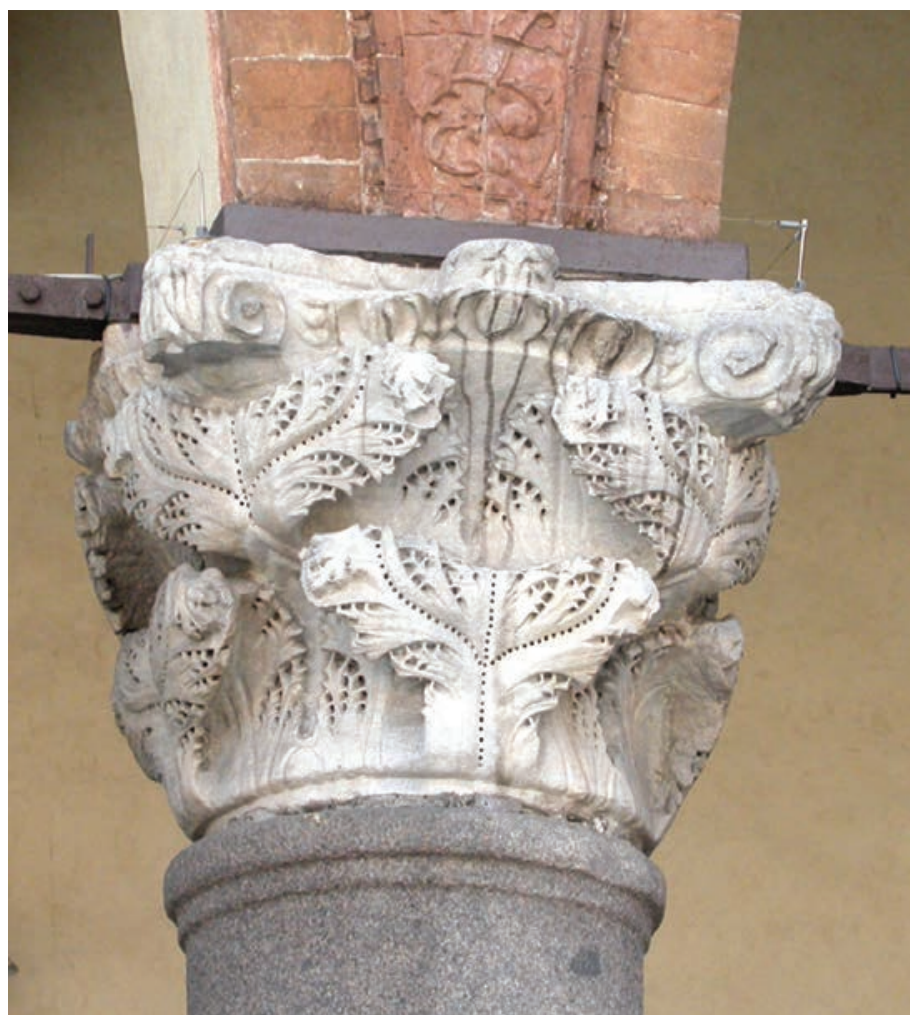

Fig.9-Ravenna, Piazza del Popolo, Palazzetto Veneziano: capitello a foglie d'acanto mosse da vento (foto autore).

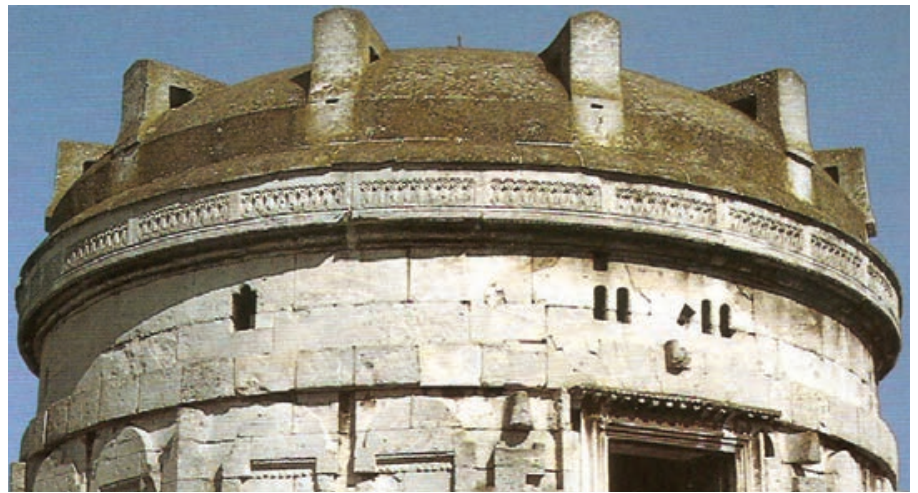

Fig.11-Ravenna, Mausoleo di Teoderico, cupola monolitica

confermano la provenienza proconnesia di tali prefabbricati, commissionati appositamente per tale edificio ${ }^{25}$.

Anche le preziose transenne, che ora delimitano l'area presbiteriale, risalgono molto probabilmente al periodo teodericiano ${ }^{26}$.

E ancora un gruppo di capitelli compositi a "foglie d'acanto mosse dal vento", originariamente appartenenti alla Ecclesia Gothorum, demolita dai Veneziani per la costruzione della rocca Brancaleone,alcuni dei quali provvisti del monogramma del re goto, sono stati reimpiegati nel portico del Palazzetto Veneziano di Piazza del Popolo a Ravenna

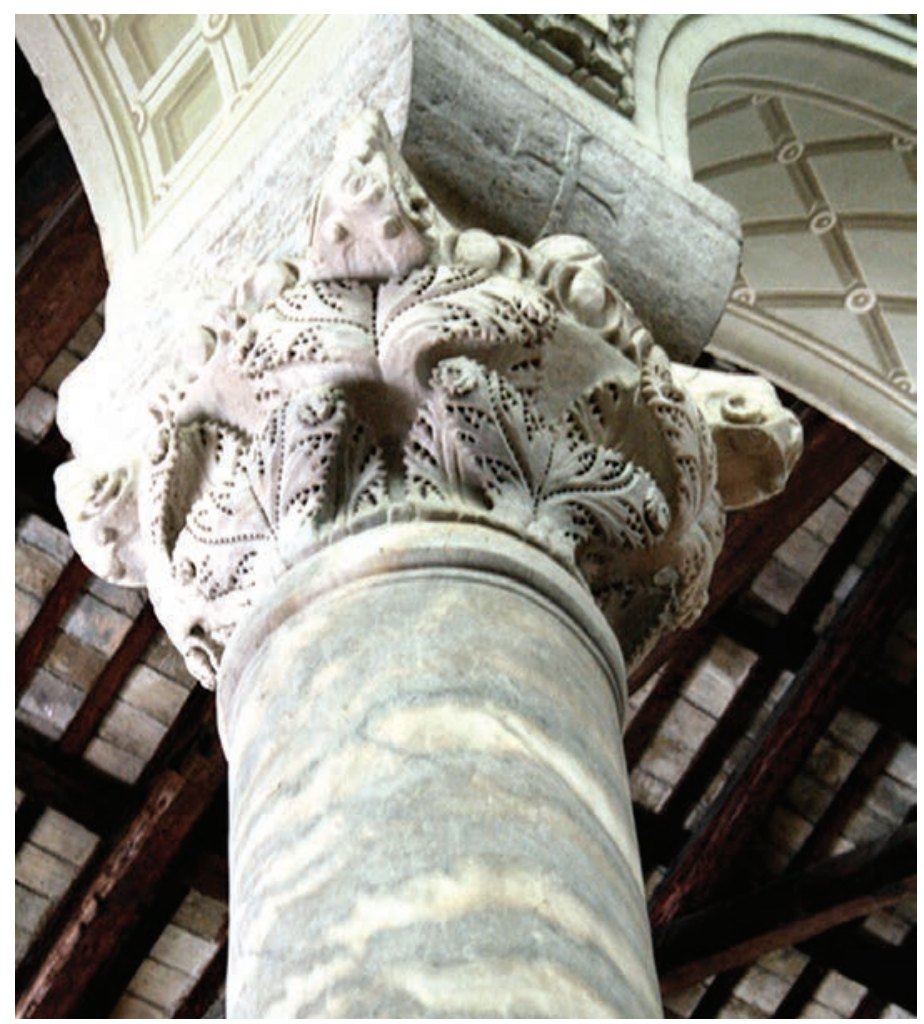

Fig. 10- Ravenna, Basilica di Sant'Apollinare in Classe: capitello a foglie d'acanto mosse dal vento

$(\text { Fig.9 })^{27}$, del tutto simili a quelli utilizzati in età giustinianea sulle ventiquattro colonne della basilica di S.Apollinare in Classe (Fig.10).

L'interesse per i materiali marmorei della capitale bizantina dovette mantenersi vivo anche nell'ambito della famiglia di Teoderico, forse dopo la sua morte, dal momento che la figlia Amalasunta e il cugino Teodato- come riferisce Cassiodoro nelle sue Variae- sollecitarono l'intervento di Giustiniano e Teodora per poter finalmente ricevere i marmi ordinati da tempo forse nella stessa Costantinopoli (Variae,X,8,2).

Contemporanemente, in età teodericiana, si assiste all'importazione della ancor più economica pietra d'Istria, come si evince dai capitelli corinzi, a due varianti presenti tuttora nella Cattedrale Ariana(oggi Chiesa dello Spirito Santo), probabilmente lavorati in loco da maestranze ravennati e dall'ambone, mirabile imitazione in pietra calcarea dei prodotti delle officine costantinopolitane o da un certo numero di sarcofagi, generalmente decorati con semplici motivi cristologici, la cui produzione continuò per tutta l'età giustinianea ${ }^{28}$.

Di pietra di aurisina(cava vicina a Trieste) sembra inoltre essere il grande monolite che sovrasta il mausoleo di Teoderico, fatto appositamente arrivare per tale singolare monumento,che costituisce un "unicum" nell'ambito dell'edilizia paleobizantina (Fig.11) ${ }^{29}$.

\footnotetext{
25 R. FARIOLI CAMPANATI, Botteghe ravennati, art. cit. (n. 19), p. 366-367.

${ }^{26}$ F. W. DEICHMANN, Ravenna Hauptstadt, 1974, op. cit. (n. 13), p. 137; R. FARIOLI CAMPANATI, Osservazioni sulla scultura del V-VI secolo, cit. (n. 17), p. 98; EADEM, Ravenna-Costantinopoli: la scultura, cart. cit. (n. 8), p. 24-25. Cfr. inoltre in partic.: B. VERNIA, L'arredo della basilica di Sant'Apollinare Nuovo a Ravenna, in: C. Rizzardi (a cura di), Venezia e Bisanzio, op. cit. (n. 8), p. 363-389, figg. 1-24.

${ }^{27}$ R. FARIOLI CAMPANATI, La scultura architettonica, art. cit. (n. 8), p. 253.

${ }^{28}$ R. FARIOLI CAMPANATI, Ravenna-Costantinopoli:la scultura, art. cit. (n. 8), p. 25; circa i sarcofagi con segni cristologici:R. FARIOLI, I sarcofagi ravennati con segni cristologici:contributo per un completamento del Corpus II, in Felix Ravenna, CXIII-IV, 1977, p. 131-159.

${ }^{29}$ R. HEIDENREICH-H. JOHANNES, Das Grabmal Theodorichs zu Ravenna, Wiesbaden 1971; C. RIZZARDI, Ravenna. Otto Monumenti patrimonio dell'umanità. L'iscrizione di Ravenna nella World Heritage Lista dell'Unesco, Ravenna 2010, p. 60-63.

$3^{30}$ Per i marchi di fabbrica vd.: F. W. DEICHMANN, Ravenna Hauptstadt des spätantiken Abendlandes, Kommentar, II, 2. Teil, Wiesbaden, 1976, p. 137-140; per i marchi dei marmorari nella capitale bizantina cfr. recentemente: A. PARIBENI, Le sigle dei marmorari e l'organizzazione del cantiere, in: A. GUIGLIA
} 

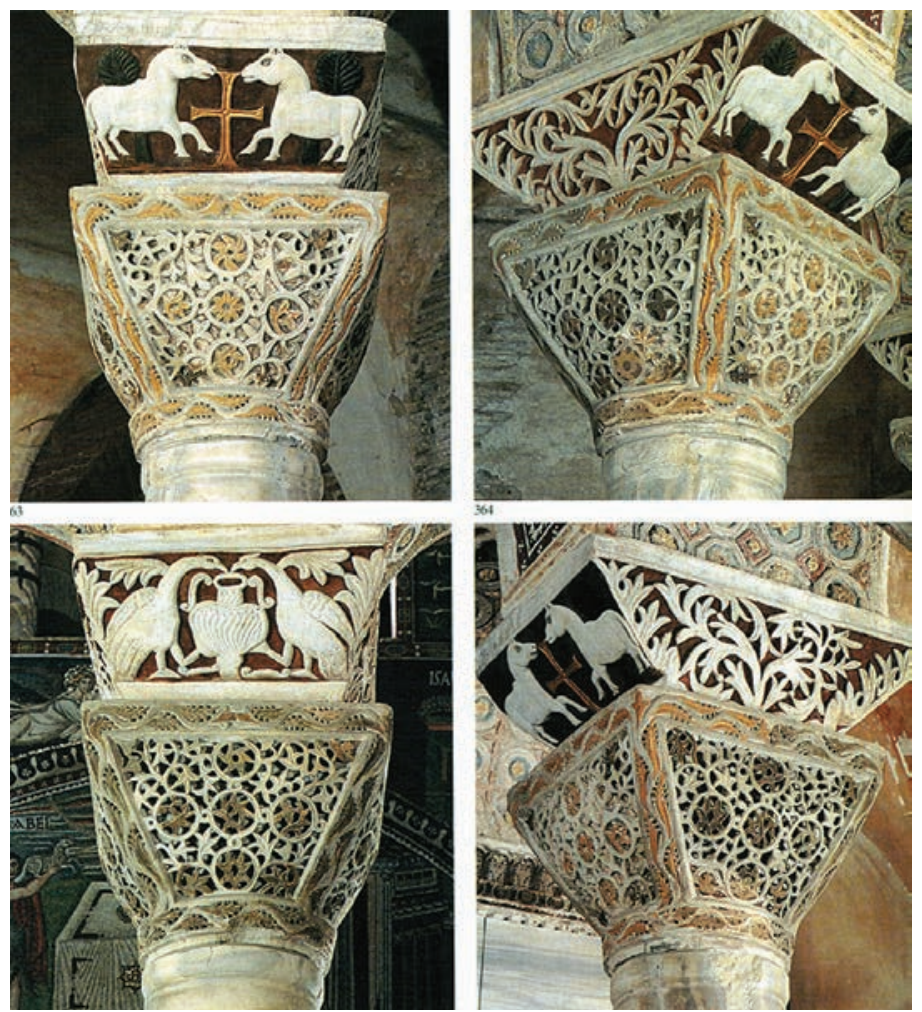

Fig. 12- Ravenna, Basilica di San Vitale, parete destra del presbiterio, trifora del pianterreno, capitello di sinistra (da: La Basilica di San Vitale, Atlante, tav. a p.207).

Il fenomeno dell'importazione di marmi lavorati continuerà e raggiungerà l'apice in età giustinianea,sia quantitativamente che qualitativamente quando, ancora dalle officine orientali del Proconneso giungeranno in abbondanza basi, capitelli,pulvini,colonne segnate da ben precisi marchi di fabbrica in lettere greche tuttora leggibili sui loro fusti ${ }^{3 \circ}$.

A Ravenna soprattutto le chiese consacrate in epoca giustinianea, S.Vitale e S.Apollinare in Classe,sono state oggetto di una particolare attenzione da parte della committenza ecclesiastica e impreziosite da sculture architettoniche e arredi marmorei di alta qualità, che trovano riscontri con le botteghe più qualificate della Metropoli ${ }^{31}$. In particolare sotto l'episcopato del vescovo Vittore (536-544) dovettero giungere i manufatti di più alto livello artistico: sontuoso doveva essere l'arredo in marmo proconnesio di San Vitale,testimoniato ancor oggi da una preziosa serie di capitelli lavorati a giorno,inseriti nell'area presbiteriale, che- sulla scia delle innovazioni proposte dagli scultori di San Polieucto- presentano un'ornamentazione avvolgente, vegetale-astratta, resa da rametti di acanto variamente combinati tali da suggerire
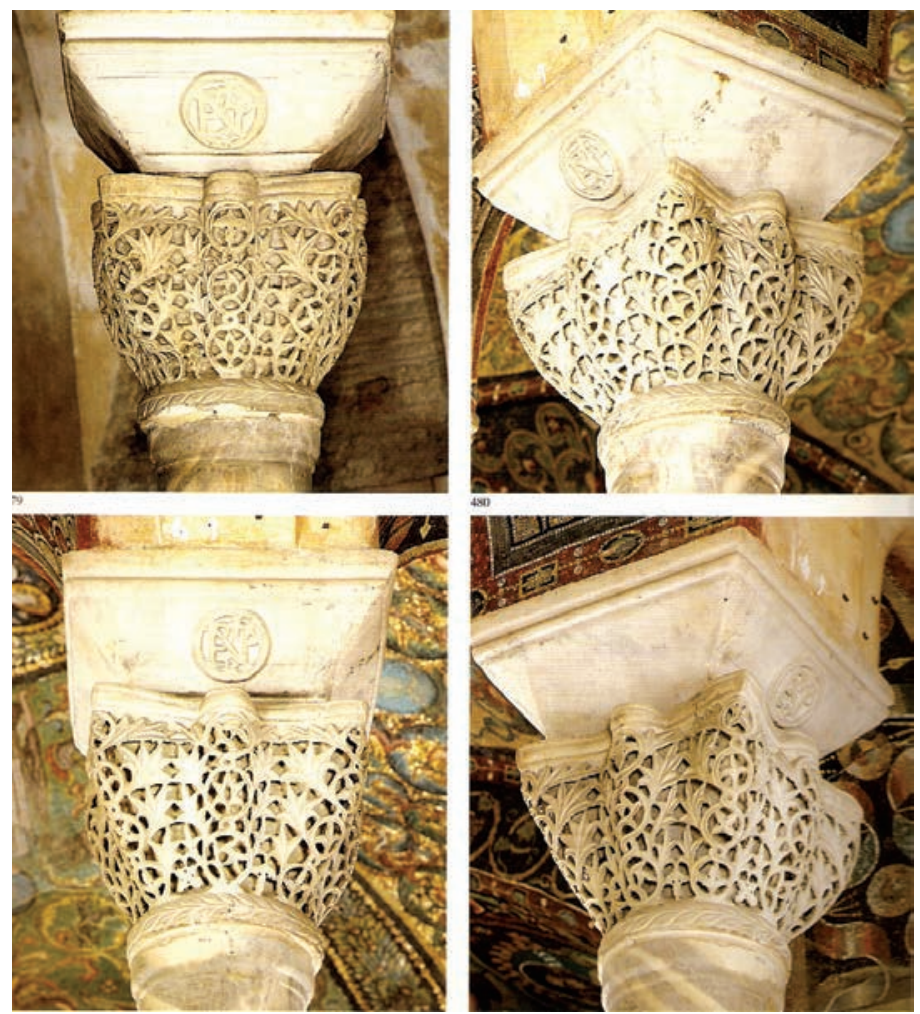

Fig.13-Ravenna, Basilica di San Vitale,parete sinistra del presbiterio, trifora a livello dei matronei: capitello increspato di sinistra (da: La Basilica di San Vitale, Atlante, tav. a p.266).

l'effetto di un merletto prezioso che annulla ogni senso di materialità o da orbicoli disposti a croce (Fig.12); fra di essi si distinguono due capitelli polilobati, in cui la superficie marmorea si dissolve in preziose increspature,producendo l'effetto di un elegante canestro(Fig.13) ${ }^{32}$; essi si collegano, per tipologia strutturale e decorativa,con i raffinati esemplari della chiesa dei Ss.Sergio e Bacco- siglati da monogrammi imperiali di Giustiniano e Teodora- frutto di una maturazione dell'innovazione artistica portata da Anicia Giuliana in San Polieucto(524-527), che sarà poi mirabilmente sintetizzata nelle sculture di Santa Sofia33.

E proprio sulla stessa scia si devono intendere i 14 capitelli che sormontano le colonne del pianterreno di San Vitale(Fig.14), che trovano un perfetto prototipo in un capitello di San Polieucto, caratterizzato da un'identica combinazione di temi geometrici e vegetali astratti di ascendenza sassanide(palmette fiancheggianti un fiore di loto),strette da un nastro "a pativ", simbolo di regalità,incorniciati da un bordo a treccia viminea che annulla ogni senso naturalistico ${ }^{34}$. Tale tipo di capitello troverà particolare diffusione in varie parti

GUIDOBALDI, C. BARSANTI, Santa Sofia di Costantinopoli. L'arredo marmoreo della Grande Chiesa giustinianea (Studi di Antichità Cristiana pubblicati a cura del Pontificio Istituto di Archeologia Cristiana, LX), Città del Vaticano 2004, p. 649-734; G. MARSILI, La committenza architettonica attraverso i marchi dei marmorari: il caso del Palazzo di Antioco a Costantinopoli, in: P. Pensabene, C. Sfameni (a cura di), La Villa restaurata e i nuovi studi sull'edilizia residenziale tardo antica, Atti del Convegno Internazionale del Centro Interuniversitario di Studi sull'Italia abitativa tardo antica nel Mediterraneo (CISEM) (Piazza Armerina 7-10 novembre 2012), Bari 2014, p. 181-189.

${ }^{31}$ Circa la scultura ravennate di epoca giustinianea cfr. R. FARIOLI CAMPANATI, Ravenna, Costantinopoli: considerazioni sulla scultura del VI secolo, in CARB, XXX, 1983, p. 205-283.

${ }^{32}$ R. FARIOLI CAMPANATI, art. cit. (n. 31), p. 206-09; EADEM, Ravenna-Costantinopoli: la scultura, art. cit. (n. 8), p. 26; cfr. inoltre: P. Angiolini Martinelli (a cura di), La Basilica di San Vitale a Ravenna, Mirabilia Italiae, 6, Modena, 1997, vol. I, Saggi e schede; vol. II Atlante fotografico.

${ }_{33}$ Cfr.: Chr. STRUBE, Polyeuktoskirche und H. Sophia (Akad. Wissenschaft), 92, München, 1984. Circa tali problematiche e confronti cfr. inoltre: E. RUSSO, Per una introduzione ai capitelli di S. Sofia di Costantinopoli, in: G. De Vergottini (a cura di), Rendiconti degli Anni 2013-2014, Accademia delle Scienze dell'Istituto di Bologna, Bononia University Press, 2015, p. 243-277, figg. 39.

${ }^{34}$ C. BARSANTI, in J. P. SODINI, Cl. BARSANTI, A. GUIGLIA GUIDOBALDI, La sculpture architecturale, art. cit. (n. 8), p. 350; R. FARIOLI, Ravenna e i suoi rapporti con Costantinopoli, art. cit. (n. 8), p. 24-226, figg. 21-23; circa la presenza di motivi sassanidi nell'arte di Ravenna cfr. C. RIZZARDI, Motivi sassanidi nell'arte di Ravenna del Ve VI secolo, in CARB, XXVIII, Ravenna, 1991, p. 367-385. 


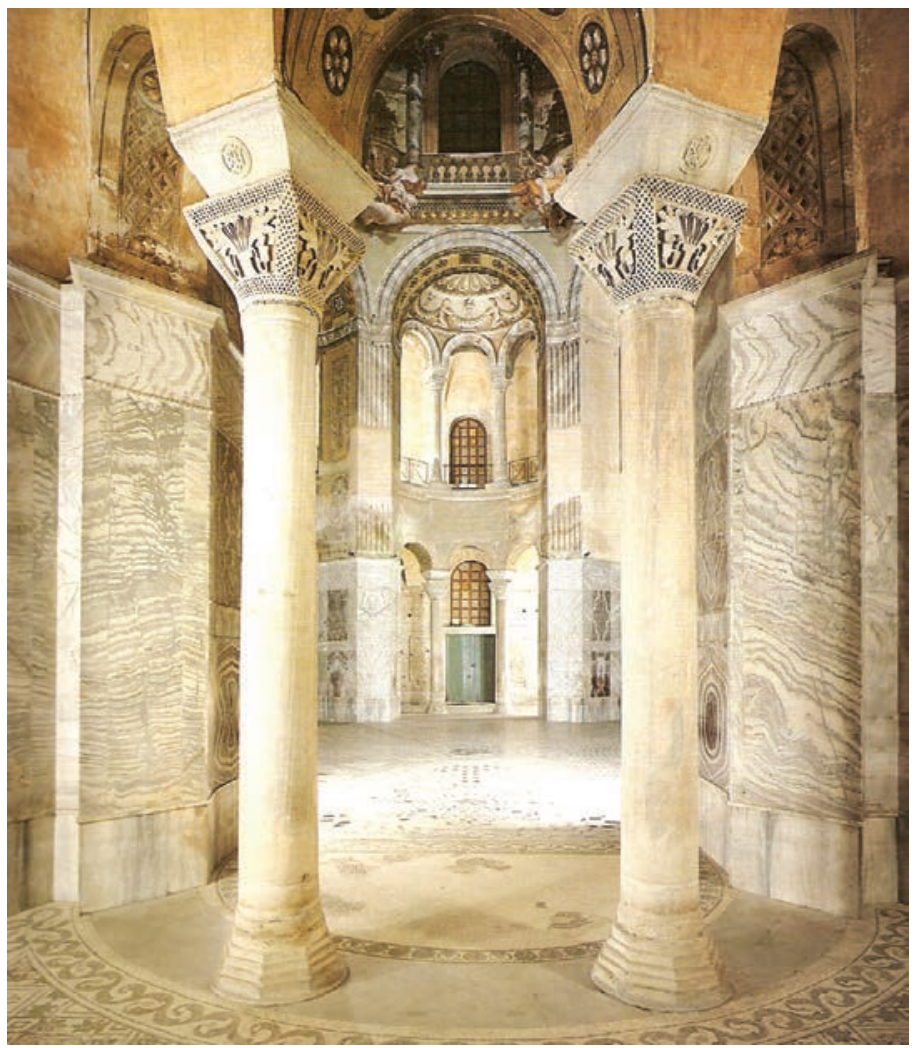

Fig.14-Ravenna, Basilica di San Vitale: colonne e capitelli del pianterreno (da: La Basilica di San Vitale, Atlante, tav. a p.110).

dell'Impero, come si riscontra a Parenzo, a Venezia,in Egitto e in Tunisia,per cui si segnala il bell'esemplare impiegato a Kairouan, nel mausoleo di Sidi el Ghariani, dimostrando l'ampiezza del fenomeno dell'esportazione dei marmi in ambito mediterraneo 35 .

Insieme con i capitelli,documentati in numerosi altri esempi(San Michele in Africisco,S.Apollinare in Classe, Museo Arcivescovile di Ravenna), giunsero a Ravenna basi e colonne per San Vitale,di marmo bianco-grigio con venature verticali, provviste di marchi di fabbrica e altre classi di marmi destinati all'arredo presbiteriale ${ }^{36}$ : oltre agli elementi architettonici di una pergula, ora conservata nel secondo chiostro di San Francesco ${ }^{37}$, sono le raffinatissime transenne lavorate a giorno che contribuiscono a dare il senso della grande perizia tecnica, raggiunta in età giustinianea,dell'amore di tutto ciò che è bello e prezioso(Figg.15,16). Tuttora conservate nel Museo Nazionale di Ravenna, esse sono decorate con motivi nastriformi, annodati in figure geometriche racchiudenti fiori,foglie,girandole, delimitate da cornici piatte o percorse da tralci vegetali, raggiungendo i più alti livelli artistici ed effetti di vibrante cromatismo ${ }^{38}$. Il raffinato corredo scultoreo,fondendosi perfettamente con

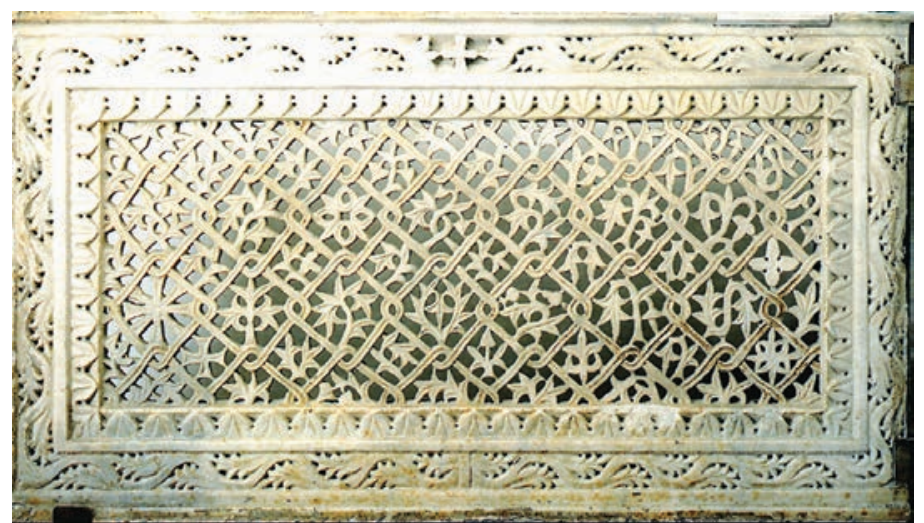

Fig.15-Ravenna, Museo Nazionale: transenna proveniente da San Vitale

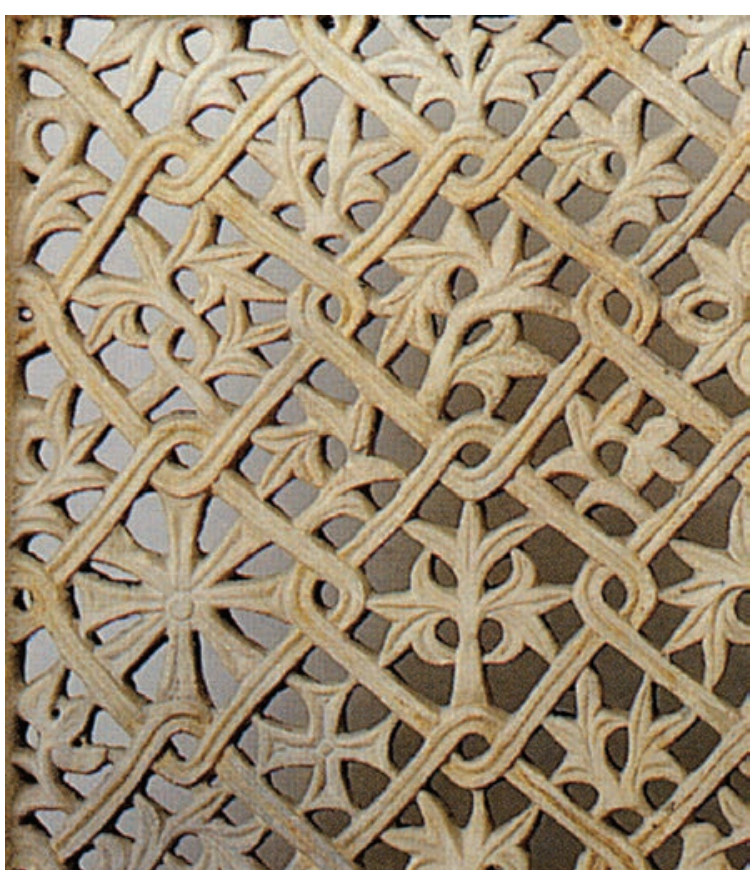

Fig.16-Ravenna, Museo Nazionale: transenna proveniente da San Vitale, particolare

l'architettura e con la decorazione musiva dorata riverberante luce, dovevano creare un ambiente metafisico magico e prezioso,uno spazio trascendente,atemporale e aspaziale,espressione di un mondo divino.Il tutto coadiuvato dalle celebrazioni liturgiche,dai paramenti degli officianti,dalle musiche e dai canti sacri,dalla complessa illuminazione.

Nello stesso Museo Nazionale si conserva un'elegante transenna proveniente da San Michele in Africisco(Fig.17), in cui vengono raggiunti gli stessi effetti decorativi di trina marmorea,mediante nastri annodati in figure geometriche circolari e quadriconche, contenenti raffinati motivi a girandola o a croce,resi mediante stilizzate foglie d'acanto ${ }^{39}$.

\footnotetext{
35 F. W. DEICHMAN, Ravenna Hauptstadt, 1976, op. cit. (n. 30), p. 96-112; per un'approfondita disamina dei capitelli di età giustinianea di Ravenna nell'ambito della produzione artistica costantinopolitana cfr. C. BARSANTI, in J. P. SODINI, Cl. BARSANTI, A. GUIGLIA GUIDOBALDI, La sculpture architecturale, art. cit. (n. 8), p. 316-336.

${ }^{36}$ F. W. DEICHMANN, Ravenna Hauptstadt, 1976, op. cit. (n. 30), p. 86-95.

${ }^{37}$ R. FARIOLI CAMPANATI, La scultura architettonica, art. cit. (n. 8), p. 258, fig. 26; C. RIZZARDI, L’impianto liturgico nelle chiese ravennati (V-VI secolo), in Hortus Artium Medievalium, v. 5, Zagreb-Motovun, 1999, p. 74, fig. 22.

${ }^{38}$ F. W. DEICHMANN, Ravenna Hauptstadt, 1989, op. cit. (n. 4), p. 324-329; C. RIZZARDI, Le transenne di San Vitale, ora nel Museo Nazionale di Ravenna, nella cultura artistica e nell'estetica bizantina, in Quaderni della Soprintendenza per i Beni Architettonici e per il Paesaggio di Ravenna(QdS), n 6, Ravenna, 2006, p. 38-51.

${ }^{39}$ F. W. DEICHMANN, Ravenna Hauptstadt, op. cit., (n. 4), p. 244, fig. 43; R. FARIOLI CAMPANATI, Ravenna-Costantinopoli: la scultura, art. cit. (n. 8), p. 27, fig. 25; P. PORTA, San Michele in Africisco a Ravenna. La scultura architettonica-decorativa, in: C. SPADONI-L. KNIFFITZ (a cura di) San Michele in Africisco e l'età giustinianea a Ravenna, Atti del Convegno "La dispora dell'arcangelo. San Michele in Africisco e l'età giustinianea". Giornate di Studio in memoria di Giuseppe Bovini (Ravenna, Sala dei mosaici, 21-22 aprile 2005), Milano , 2007, p. 200-201, tav. 24.
} 


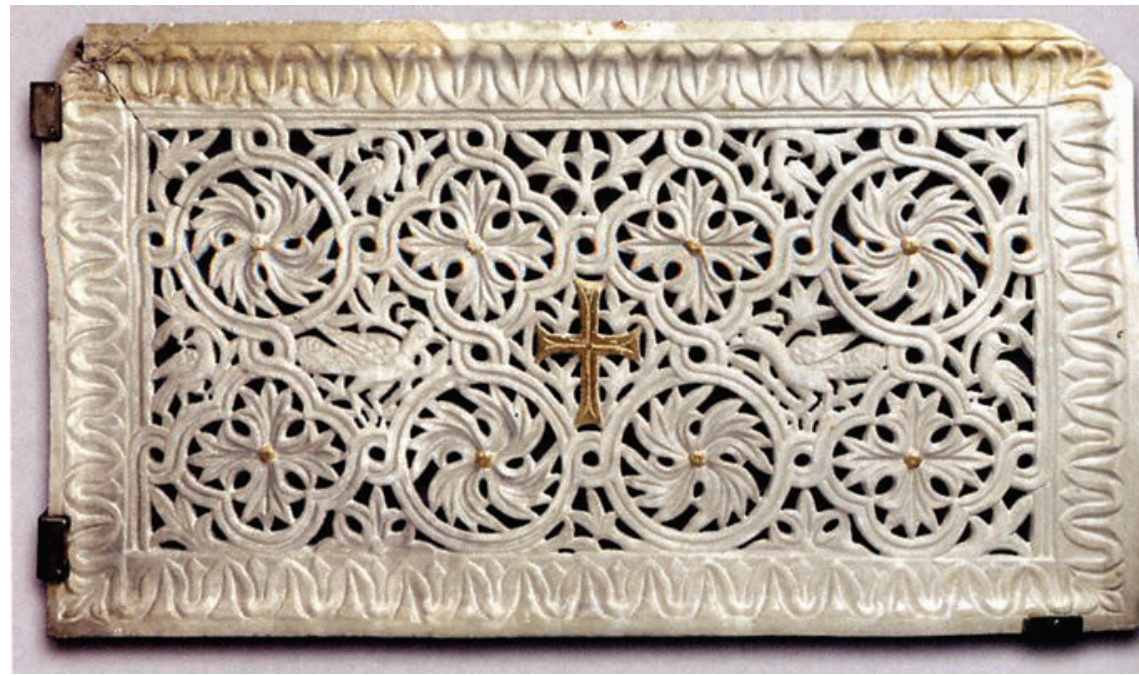

Fig.17- Ravenna, Museo Nazionale: transenna proveniente da San Michele in Africisco
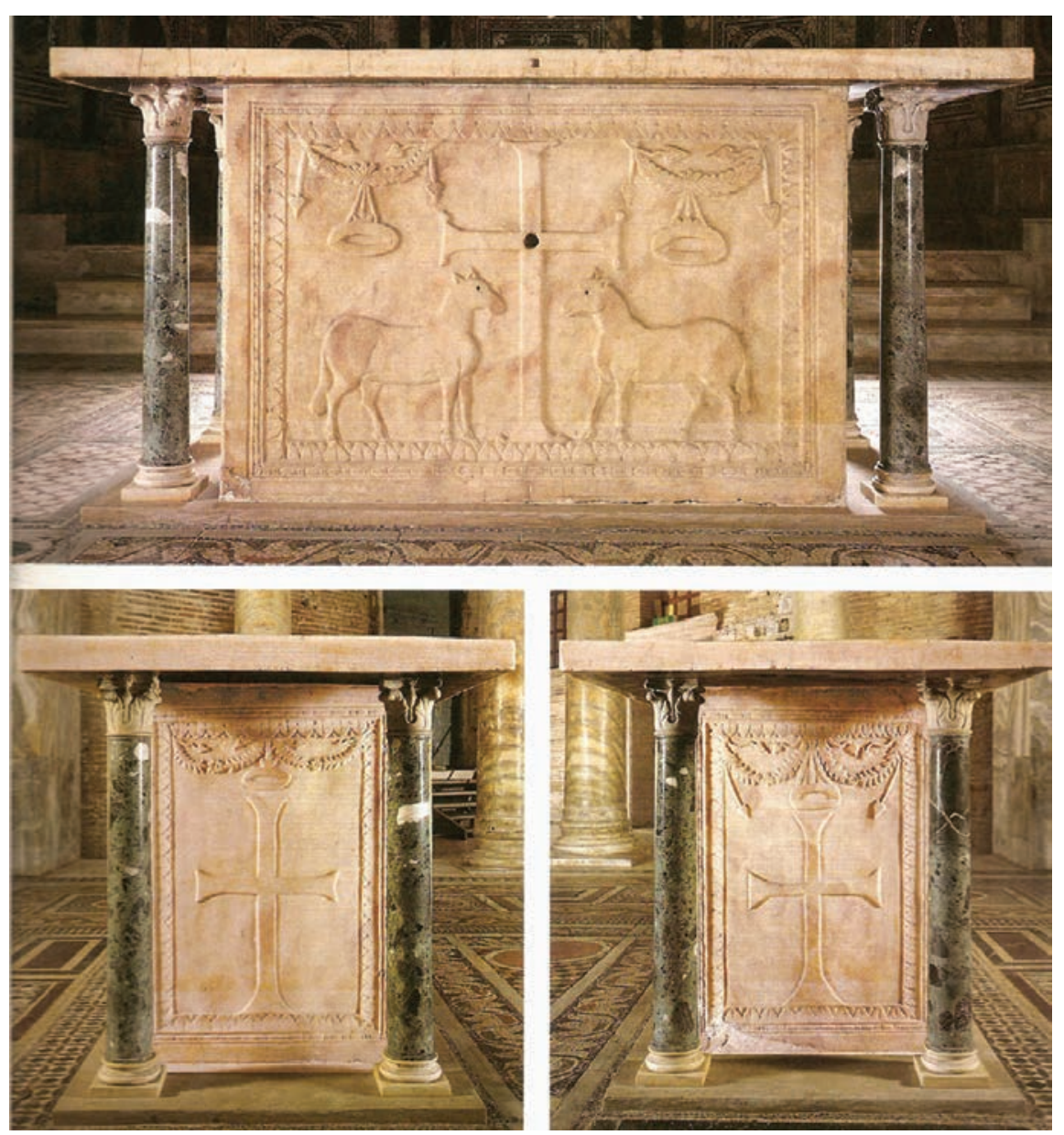

Fig.18- Ravenna, Basilica di San Vitale: altare a cassa, fronte (in alto); lati (in basso) (da: La Basilica di San Vitale, Atlante, tav. a p. 203)

Esemplari analoghi, ora nel Museo Arcivescovile,furono impiegati anche per la risistemazione e conseguente abbellimento della basilica Ursiana, in epoca giustinianea ${ }^{40}$.
Ravenna possiede quindi un notevole patrimonio di tali lastre traforate che, data la loro fragilità, sono andate altrove in gran parte distrutte; anche a Costantinopoli ne restano poche, anche se recenti indagini hanno portato alla scoperta di nuovi esemplari: tre tuttora riutilizzate alle estremità orientali delle gallerie di S.Sofia prospicienti il presbiterio,altre riscontrate nel corridoio della loggia imperiale ottomana del pianterreno e due riutilizzate nel nimbar ottomano dei Ss. Sergio e Bacco, a testimonianza dell'input culturale dato anche in questo caso dalla capitale bizantina ${ }^{41}$.

Quanto all'arredo liturgico di San Vitale,particolarmente importante è l'altare a cassa, posto al centro del presbiterio e ricomposto nel 1954(Fig.18): esso presenta una mensa in alabastro,sorretta da 4 colonnine in verde di Tessaglia ed una decorazione simbolica, costituita da agnelli affrontati ad una croce,sia sulla lastra anteriore che sulle due laterali,di marmo proconneso, presumibilmente fatte arrivare da Costantinopoli ${ }^{42}$. Impossibile è ricordare tutti i manufatti marmorei arrivati a Ravenna nel V-VI secolo,per cui rimando ai tanti esaustivi e particolareggiati studi esistenti in merito. Mi limiterò a tal punto, alla luce di quanto esposto, a fare alcune considerazioni.

Sembra ormai indubitabile che, dopo il trasferimento della capitale a Ravenna, grazie anche alla presenza di un porto commerciale,si sia provveduto a commissioni sistematiche di manufatti scultorei, talora in gruppi omogenei,il cui impiego doveva essere richiesto nella nuova edilizia civile e soprattutto religiosa,in seguito al trasferimento della corte. Il tutto dovette essere conseguente non solo a motivi economici e di traffici commerciali, ma anche a ragioni ideologiche e politiche ben precise, alla necessità di instaurare stretti rapporti fra le due capitali e di rafforzare il potere centrale, specie in età giustinianea.

Una committenza privilegiata, costituita da Imperatrici, re, imperatori, ma anche da vescovi,o membri della più alta aristocrazia dovette preoccuparsi di sostenere tale scambio,foriero di ben più strette intese e in ogni caso dimostrando il carattere elitario assunto nel V-VI secolo dall'impiego di tali preziosi materiali. Ciò viene confermato anche dal protostorico A.Agnello nel suo Liber Pontificalis Ecclesiae Ravennatis(IX secolo), che ricorda come l'élite ecclesiastica ravennate si riforniva di marmi proprio nell'area costantinopolitana: lo stesso arcivescovo Massimiano di Pola- creatura

\footnotetext{
$\overline{4^{\circ}}$ F. W. DEICHMANN, Ravenna Hauptstadt, 1989, op. cit. (n. 4), p. 324-329.

${ }^{41}$ Circa le transenne bizantine già note o recentemente riscoperte in Santa Sofia cfr. :A. GUIGLIA GUIDOBALDI, Una ricerca coordinata sull'arredo marmoreo di età giustinianea della Santa Sofia di Costantinopoli, Atti del VI Congresso Nazionale di Studi Bizantini(Catania-Messina 200o), Catania 2004, p. 397-428; A. GUIGLIA GUIDOBALDI, C. BARSANTI, M. DELLA VALLE, R . FLAMINIO, A. PARIBENI, A. BILBAN YALÇIN, La collezione delle sculture bizantine nel Museo della Santa Sofia a Istanbul, in RolSA IV, Rivista on line di Storia dell'Arte. Dipartimento di Storia dell'Arte. Università di Roma, IV, 2005, pp. 1-11, figg. 20-22, 28-31.

${ }^{42}$ L. SOTIRA, op. cit. (n. 15), p. 53-58.
} 


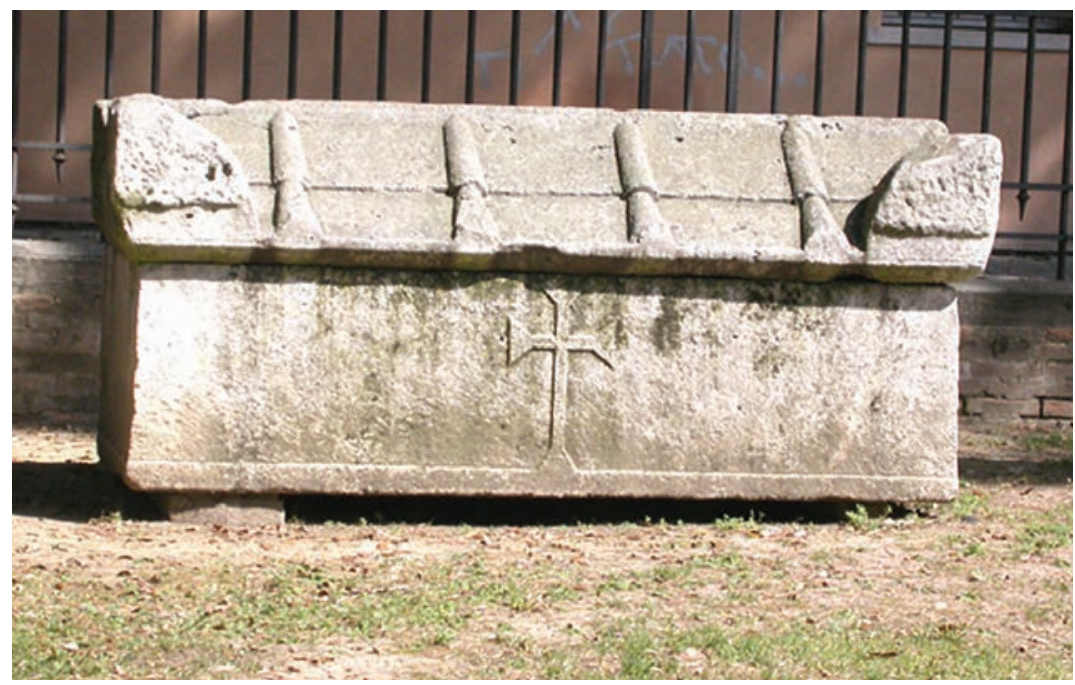

Fig. 19- Ravenna, prato di San Vitale: sarcofago in pietra istriana decorato con croce latina (fine VI sec.) (foto autore).

di Giustiniano, sempre in linea con la politica imperiale- si sarebbe ivi procurato i materiali per la scomparsa basilica di S.Stefano, da lui fatta innalzare, e marmi e colonne del Proconneso per la chiesa di Sant'Andrea ${ }^{43}$.

Si instaura quindi,in linea con la politica imperiale,una dinamica circolazione di arte e cultura fra l'Impero d'Oriente e d'Occidente, che trova in Ravenna un punto di riferimento speciale.

Tale vasta e grossa operazione dovette richiedere, nella stessa Ravenna, una serie di strutture capaci di accogliere i materiali importati, in attesa di essere utilizzati o talora rifiniti.

Si è quindi ipotizzata l'esistenza di una statio marmoru$m$,capace di accogliere i marmi importati, facente capo ad un funzionario che amministrava e gestiva la distribuzione di tali materiali, commercializzandoli o assegnandoli ad officine locali, come pare dedursi da una lettera di Cassiodoro(Variae,III,1), secondo cui il re Teoderico si rivolge a un certo Daniel, raccomandandosi di non alzare il prezzo dei marmi e dei sarcofagi in particolare ${ }^{44}$.

L'ubicazione di uno di tali magazzini di stoccaggio si può ipotizzare presso la chiesa di San Giovanni Battista-situata davanti a porta Guarcinorum(S. Vittore), attuale porta Serrata, nei pressi di San Vitale e del flumen Padenna- che fu consacrata da Massimiano e denominata ad Marmorata ${ }^{45}$.

Si è anche cercato di calcolare la quantità, o meglio il peso del materiale marmoreo giunto a Ravenna nel periodo compreso fra l'inizio dell'età teodericiana e la fine dell'età giustinianea(fineV-fine VI sec.),corrispondente a 1.556 tonnellate,raggiungendo la massima quantità per la chiesa di S.Vitale, corrispondente a circa 188 tonnellate ${ }^{46}$.

Dopo la fine dell'età giustinianea,ridotti i commerci internazionali,quasi scomparsa l'importazione dei preziosi marmi del Proconneso, una nuova ondata di materiale in pietra d'Istria e in pietra calcarea proveniente dall'isola di Brač- usato per sarcofagi decorati a motivi simbolici e prevalentemente a semplici croci(Fig.19)- testimonia per Ravenna una fase di maggior distacco da Costantinopoli, quindi di più limitati orizzonti geografici e culturali e forse di maggiori ristrettezze economiche, con conseguente necessità di rivolgersi a lidi più vicini ${ }^{47}$.

A questo punto un nuovo capitolo si potrebbe aprire con il problema delle maestranze: marmi lavorati a Costantinopoli o nelle cave di origine,oppure marmi lavorati a Ravenna da scultori di provenienza costantinopolitana o locale? Il dibattito, che ha coinvolto e diviso vari studiosi in diversi periodi, è quanto mai controverso e ancora acceso,ma di ciò si tratterà in altra sede.

43 AGNELLUS, Liber Pontificalis Ecclesiae Ravennatis, XXVI, 73 e 76; Y. MARANO, op. cit. (n. 8), p. 164.

44 P. PENSABENE, C. BARSANTI, Reimpiego e importazione di marmi, cit. (n. 8), p. 464.

45. J. G. HARPER, art. cit. (n. 8), p. 134; per la chiesa di S. Giovanni Battista in Marmorata cfr. :F. W. DEICHMANN, Ravenna Hauptstadt, 1976, op. cit. (n. 30), p. 331.

${ }^{46} \mathrm{Vd}$. a tal proposito lo studio di J. G. HARPER, art. cit. (n. 8).

${ }_{47}$ R. FARIOLI, La scultura architettonica, art. cit. (n. 8), p. 264; Y. A. MARANO, op. cit. (n. 8), p. 171. Per i sarcofago di San Vitale in pietra calcarea(con coperchio pagano) cfr.: Cfr. C. RIZZARDI, I sarcofagi conservati in San Vitale di Ravenna e nel prato circostante, in: P. Angiolini Martinelli (a cura di), La Basilica di San Vitale a Ravenna, op. cit. (n. 32), scheda n. 48. Per i sarcofagi in pietra calcarea prodotti in Istria e Dalmazia vd.: N. CAMBI, I sarcofagi della tarda Antichità in Istria e Dalmazia, in: F. Bisconti e H. Brandenburg(a cura di), Sarcofagi tardo antichi, paleocristiani e altomedievali, op. cit. (n. 17), p. 7796. Per un catalogo aggiornato della scultura istriana cfr. la recente opera: J. MATEJČIĆ, S. MUSTAČ, Scultura dal IV al XIII secolo (schede di P. Chevalier, M. Jurković), Parenzo, 2014. 